\title{
Prevalence of Olfactory or Gustatory Dysfunction in COVID-19 Patients: An Analysis Based on Korean Nationwide Claims Data
}

\author{
Eun-Jin $\mathrm{Ahn}^{1}$ iD $\cdot$ Hyun Jin $\mathrm{Min}^{2}$ (iD \\ Departments of ${ }^{1}$ Anesthesiology and Pain Medicine and ${ }^{2}$ Otorhinolaryngology-Head and Neck Surgery, Chung-Ang University Medical Center, \\ Chung-Ang University College of Medicine, Seoul, Korea
}

Coronavirus disease 2019 (COVID-19), caused by severe acute respiratory syndrome coronavirus 2 , has become one of the most important threats to human health [1]. The clinical manifestations of COVID-19 range from asymptomatic status to severe life-threatening systemic symptoms. Interestingly, olfactory dysfunction has been reported in COVID-19, and when combined with other symptoms, it appears to be a strong predictor of COVID-19 [2]. However, a significant proportion of COVID-19 patients reporting olfactory dysfunction do not exhibit it on objective testing [3].

The South Korean (hereafter, Korean) government has provided a COVID-19 dataset containing data on healthcare service use from the Health Insurance Review and Assessment Service of Korea (HIRA). In Korea, approximately $97 \%$ of the population is covered under the Korean National Health Insurance Service, and the HIRA reviews almost all medical information [4]. However, no study has yet evaluated COVID-19 patients with olfactory or gustatory dysfunction using the HIRA dataset. We therefore aimed to evaluate the prevalence and characteristics of COVID-19 patients with olfactory or gustatory dysfunction through a retrospective analysis of the cases recorded in the HIRA COVID-19 dataset.

This study was approved by the Institutional Review Board of Chung-Ang University Hospital (IRB No. 2004-010-19310), and the requirement for informed consent was waived. Data were extracted on May 15, 2020, from the HIRA COVID-19 dataset

\footnotetext{
- Received October 30, 2020

Revised December 15, 2020

Accepted January 5, 2021

- Corresponding author: Hyun Jin Min

Department of Otorhinolaryngology-Head and Neck Surgery, Chung-Ang University College of Medicine, 84 Heukseok-ro, Dongjak-gu, Seoul 06974, Korea

Tel: +82-2-6299-1765, Fax: +82-2-825-1765

E-mail: jjinient@gmail.com
}

(https://hira-covid19.net), prepared by a collaboration between the Ministry of Health and Welfare of Korea and the HIRA. Dataset 1 contained claims data with a classification code of MT043 (medical cost support due to national disaster), based on the serial number of the claims statement, that contained the D6584 code (real-time polymerase chain reaction), COVID-19-related disease codes (B342, B972, Z208, Z290, U18, U181, Z038, Z115, U071, and U072), and other COVID-19-related fee codes (for COVID-19-related admission fees, management fees, intrauterine devices, isolation, public relief hospitals, residential treatment centers, screening centers, and negative pressure rooms). Dataset 2 contained healthcare service use data on claims for healthcare service use from January 2017 to May 15, 2020 for patients who had submitted claims for the above-mentioned COVID-19-related criteria at least once. There were 234,427 persons of COVID19-related claim submissions, corresponding to 476,508 separate claim cases. Among the 11,018 confirmed COVID-19 cases, we obtained claims statements for 7,590 patients after matching the two datasets.

We included all cases of COVID-19 patients in the HIRA dataset diagnosed before May 15, 2020. Patients were categorized as having olfactory or gustatory dysfunction based on a principal diagnosis of anosmia (R430), parosmia (R431), taste disorder (R432, R438), mixed disorder (R438), or smell disorder (R430, R431, R438) according to International Classification of Diseases, 10th Revision, Clinical Modification (ICD-10-CM) codes. Patients with olfactory or gustatory dysfunction after a confirmed COVID-19 diagnosis were included from Dataset 1. Patients with olfactory or gustatory dysfunction in the 1-month period before the diagnosis of COVID-19 were included from Dataset 2.

Patient characteristics including sex, age, comorbidities, intensive care unit (ICU) stay, ventilator care, and death were recorded. Comorbidities included a history of allergic rhinitis (J30), chronic rhinitis (J31), acute sinusitis (J01), chronic sinusitis (J32),

Copyright (C) 2021 by Korean Society of Otorhinolaryngology-Head and Neck Surgery.

This is an open-access article distributed under the terms of the Creative Commons Attribution Non-Commercial License (https://creativecommons.org/licenses/by-nc/4.0) which permits unrestricted non-commercial use, distribution, and reproduction in any medium, provided the original work is properly cited. 
Table 1. Age and sex distribution of COVID-19 patients with or without olfactory/gustatory dysfunction

\begin{tabular}{|c|c|c|c|}
\hline Variable & With olfactory/gustatory dysfunction & Without olfactory/gustatory dysfunction & $P$-value \\
\hline & Anosmia $(n=36)$ & Without dysfunction $(n=7,554)$ & \\
\hline Sex (male:female) & $12: 24$ & $3,083: 4,471$ & 0.362 \\
\hline \multirow[t]{2}{*}{ Age (yr) } & $36(24-36)$ & $52(29-65)$ & $<0.001^{*}$ \\
\hline & Parosmia $(n=2)$ & Without dysfunction $(n=7,598)$ & \\
\hline Sex (male:female) & $1: 1$ & $3,094: 4,494$ & 0.791 \\
\hline \multirow[t]{2}{*}{ Age (yr) } & $25(24-26)$ & $47(27-60)$ & 0.114 \\
\hline & Taste $(n=5)$ & Without dysfunction $(n=7,585)$ & \\
\hline Sex (male:female) & $2: 3$ & $3,093: 4,492$ & 0.675 \\
\hline \multirow[t]{2}{*}{ Age $(y r)$} & $25(24-26)$ & $47(27-60)$ & 0.119 \\
\hline & Mixed $(n=4)$ & Without dysfunction $(n=7,586)$ & \\
\hline Sex (male:female) & $2: 2$ & $3,093: 4,493$ & 0.894 \\
\hline \multirow[t]{2}{*}{ Age (yr) } & $31(23-43.5)$ & $47(27-60)$ & 0.546 \\
\hline & Total $(n=43)$ & Without dysfunction $(n=7,547)$ & \\
\hline Sex (male:female) & $15: 28$ & $3,080: 4,467$ & 0.527 \\
\hline Age (yr) & $27(24-36)$ & $47(27-60)$ & $<0.001^{*}$ \\
\hline
\end{tabular}

Values are presented as number or median (interquartile range).

COVID-19, coronavirus disease 2019.

${ }^{\star} P<0.05$ for a comparison between two groups.

and septal deviation (J342), as defined by ICD-10-CM codes from Dataset 2. The primary outcome was the prevalence of olfactory or gustatory dysfunction among confirmed COVID-19 patients.

For continuous variables, data were presented as median (range), and groups were compared using the Mann-Whitney $U$-test. Descriptive variables were analyzed using the chi-square test or Fisher exact test, as appropriate. Categorical variables were presented as absolute numbers (percentages). A $P$-value $<0.05$ was considered to indicate statistical significance. SAS ver. 9.3 (SAS Institute, Cary, NC, USA) was used for analyses. All statistical tests were two-sided with a significance level of 0.05 .

We identified 7,590 patients with confirmed COVID-19 before May 15, 2020. Among them, 32 had reported olfactory or gustatory dysfunction in the 1-month period prior to their COVID-19 diagnosis; these patients were diagnosed with anosmia. Additionally, 11 patients were diagnosed with olfactory or gustatory dysfunction during COVID-19 treatment (4, 2, 5, and 4 patients with anosmia, parosmia, taste disorder, and mixed disorder, respectively). Overall, 43 patients were diagnosed with olfactory or gustatory dysfunction $(36,2,5$, and 4 patients with anosmia, parosmia, taste disorder, and mixed disorder, respectively) (Supplementary Fig. 1). When comparing patients with and without olfactory or gustatory dysfunction, the proportion of men was not significantly different between the two groups $(P=0.43)$. However, age differed significantly between the two groups (27 years [range, 24-36 years] vs. 47 years [range, 27-60 years], $P<0.001$ ); the median age was significantly lower in patients with olfactory or gustatory dysfunction (Table 1).

Regarding the prevalence of sinonasal diseases, COVID-19 patients with olfactory or gustatory dysfunction demonstrated a significantly higher prevalence of allergic rhinitis, septal deviation, chronic rhinitis, and acute sinusitis than those without olfactory or gustatory dysfunction (Table 2).

The presence of extra-respiratory manifestations of COVID19 could be associated with its prognosis [5]. We found that mean age was significantly lower in COVID-19 patients with olfactory or gustatory dysfunction, and none of these patients died, underwent ICU treatment, or received mechanical ventilation (data not shown). Another study reported that patients with olfactory dysfunction were significantly younger and showed a milder course of the disease than patients without olfactory dysfunction [6]. Further studies are needed to investigate COVID-19 progression according to the presence of olfactory or gustatory dysfunction.

Our study's major limitation is that the patients were selected according to the diagnoses registered in the HIRA datasets. Upon reviewing the HIRA datasets of COVID-19 patients, we noted a lower prevalence of olfactory or gustatory dysfunction than has been observed among patients in other countries [7]. This might have been due to incomplete registration by doctors because they were unsure whether the patients had true symptoms, as a conventional olfactory function testing was not applicable for patients with a suspected COVID-19 diagnosis. Although the HIRA data are very unique and valuable, there is a possibility of up- or down-coding of diseases, and the registration of comorbidities could be affected by the doctor's intentions. Since the Korea National Health Insurance Service is unique and the datasets based on this system contain valuable data, we recommend that concurrent medical conditions or suspected diseases be registered carefully in this system to facilitate future investigations. 
Table 2. Prevalence of sinonasal diseases in patients with olfactory/gustatory dysfunction

\begin{tabular}{|c|c|c|c|}
\hline Variable & With olfactory/gustatory dysfunction & Without olfactory/gustatory dysfunction & $P$-value \\
\hline & Anosmia $(n=36)$ & Without dysfunction $(n=7,554)$ & \\
\hline Allergic rhinitis & $35(97.2)$ & $6,006(79.1)$ & $0.009^{*}$ \\
\hline Chronic rhinitis & $7(19.4)$ & $817(10.7)$ & 0.097 \\
\hline Septal deviation & $6(16.6)$ & $284(3.74)$ & $<0.001^{*}$ \\
\hline Chronic sinusitis & $16(44.4)$ & $1,275(16.8)$ & $<0.001^{*}$ \\
\hline \multirow[t]{2}{*}{ Acute sinusitis } & $17(47.2)$ & $1,938(25.5)$ & $0.003^{*}$ \\
\hline & Parosmia $(n=2)$ & Without dysfunction $(n=7,588)$ & \\
\hline Allergic rhinitis & $2(100)$ & $6,006(79.1)$ & 1.000 \\
\hline Chronic rhinitis & 0 & $817(10.7)$ & 1.000 \\
\hline Septal deviation & 0 & $284(3.74)$ & 1.000 \\
\hline Chronic sinusitis & 0 & $1,275(16.8)$ & 1.000 \\
\hline \multirow[t]{2}{*}{ Acute sinusitis } & 0 & $1,938(25.5)$ & 1.000 \\
\hline & Taste $(n=5)$ & Without dysfunction $(n=7,585)$ & \\
\hline Allergic rhinitis & $5(100)$ & $6,006(79.1)$ & 0.551 \\
\hline Chronic rhinitis & 0 & $817(10.7)$ & 1.000 \\
\hline Septal deviation & $1(20)$ & $284(3.7)$ & 0.056 \\
\hline Chronic sinusitis & $3(60)$ & $1,275(16.8)$ & $0.009^{*}$ \\
\hline \multirow[t]{2}{*}{ Acute sinusitis } & $2(40)$ & $1,938(25.5)$ & 0.819 \\
\hline & Mixed $(n=4)$ & Without dysfunction $(n=7,586)$ & \\
\hline Allergic rhinitis & $4(100)$ & $6,006(79.1)$ & 0.682 \\
\hline Chronic rhinitis & 0 & $817(10.7)$ & 1.000 \\
\hline Septal deviation & $1(25)$ & $284(3.7)$ & 0.056 \\
\hline Chronic sinusitis & $3(75)$ & $1,275(16.8)$ & $0.009^{*}$ \\
\hline \multirow[t]{2}{*}{ Acute sinusitis } & $2(50)$ & $1,938(25.5)$ & 0.584 \\
\hline & Total $(n=43)$ & Without dysfunction $(n=7,547)$ & \\
\hline Allergic rhinitis & $42(97.7)$ & $6,006(79.1)$ & $0.003^{*}$ \\
\hline Chronic rhinitis & $7(16.3)$ & $817(10.7)$ & 0.368 \\
\hline Septal deviation & $7(16.3)$ & $284(3.74)$ & $<0.001^{*}$ \\
\hline Chronic sinusitis & $19(44.2)$ & $1,275(16.8)$ & $<0.001^{*}$ \\
\hline Acute sinusitis & $19(44.2)$ & $1,938(25.5)$ & $0.006^{*}$ \\
\hline
\end{tabular}

Values are presented as number (\%).

${ }^{*} P<0.05$ for a comparison between two groups.

\section{CONFLICT OF INTEREST}

No potential conflict of interest relevant to this article was reported.

\section{ORCID}

Eun-Jin Ahn https://orcid.org/0000-0001-6321-5285

Hyun Jin Min https://orcid.org/0000-0003-3075-1350

\section{AUTHOR CONTRIBUTIONS}

Conceptualization: HJM. Data curation, Formal analysis, Methodology, \& Project administration: all authors. Visualization: HJM. Writing-original draft: all authors. Writing-review \& editing: HJM.

\section{SUPPLEMENTARY MATERIALS}

Supplementary material can be found via https://doi.org/10. 21053/ceo.2020.02215.

\section{REFERENCES}

1. Bousquet J, Akdis C, Jutel M, Bachert C, Klimek L, Agache I, et al. Intranasal corticosteroids in allergic rhinitis in COVID-19 infected patients: an ARIA-EAACI statement. Allergy. 2020 Mar 31 [Epub]. https://doi.org/10.1111/all.14302.

2. Eliezer M, Hautefort C, Hamel AL, Verillaud B, Herman P, Houdart $\mathrm{E}$, et al. Sudden and complete olfactory loss of function as a possible symptom of COVID-19. JAMA Otolaryngol Head Neck Surg. 2020 Jul;146(7):674-5.

3. Lechien JR, Cabaraux P, Chiesa-Estomba CM, Khalife M, Hans S, Calvo-Henriquez C, et al. Objective olfactory evaluation of self-reported loss of smell in a case series of 86 COVID-19 patients. Head Neck. 2020 July;42(7):1583-90. 
4. Park YJ, Kim JM, Lee BR, Kim TH, Lee EG. Annual prevalence and economic burden of genital warts in Korea: Health Insurance Review and Assessment (HIRA) service data from 2007 to 2015. Epidemiol Infect. 2018 Jan;146(2):177-86.

5. Lai CC, KoWC, Lee PI, Jean SS, Hsueh PR. Extra-respiratory manifestations of COVID-19. Int J Antimicrob Agents. 2020 Aug;56(2): 106024.
6. Sanli DE, Altundag A, Kandemirli SG, Yildirim D, Sanli AN, Saatci O, et al. Relationship between disease severity and serum IL-6 levels in COVID-19 anosmia. Am J Otolaryngol. 2021 Jan-Feb;42(1):102796. 7. Tong JY, Wong A, Zhu D, Fastenberg JH, Tham T. The prevalence of olfactory and gustatory dysfunction in COVID-19 patients: a systematic review and meta-analysis. Otolaryngol Head Neck Surg. 2020 Jul;163(1):3-11. 